Pacific

Journal of

Mathematics

\title{
STRONGLY POSITIVE REPRESENTATIONS \\ OF EVEN GSPIN GROUPS
}

YEANSU KIM 


\title{
STRONGLY POSITIVE REPRESENTATIONS OF EVEN GSPIN GROUPS
}

\author{
YEANSU KIM
}

\begin{abstract}
We obtain a classification of strongly positive representations of split even general spin groups over a $\boldsymbol{p}$-adic field $\boldsymbol{F}$ using the Jacquet module method (Tadić's structure formula). Furthermore, we study discrete series representations of split even general spin groups over $F$.
\end{abstract}

\section{Introduction}

The classifications of (strongly positive) discrete series representations of metaplectic groups, classical groups, and odd GSpin groups over a $p$-adic field have been studied by several authors [Kim 2015b; Matić 2011; Mœglin 2002; Mœglin and Tadić 2002; Zelevinsky 1980]. The main purpose of this paper is to obtain a classification of strongly positive representations of split even GSpin groups over a nonarchimedean local field $F$ of characteristic different from two, assuming the uniqueness of the nonnegative rank one reducibility point (see Remark 1.2 for more details about this assumption). Our results generalize Matić's algebraic approach [Matić 2011] to the case of even GSpin groups. Our results for even GSpin groups can be applied to even special orthogonal groups to classify strongly positive representations of $\mathrm{SO}_{2 n}$. In addition, the results are parallel to those for odd GSpin groups [Kim 2015b]. However, parts of their proofs are quite different because of differences in the group structures. For example, there are two nonconjugate standard parabolic subgroups whose Levi subgroups are of the form $\mathrm{GL}_{n_{1}} \times \mathrm{GL}_{n_{2}} \times \cdots \times \mathrm{GL}_{n_{k}}$ in the even case; therefore, we classify $D\left(\rho ; \sigma_{\text {cusp }}, c\left(\sigma_{\text {cusp }}\right)\right)$ instead of $D\left(\rho ; \sigma_{\text {cusp }}\right)$ in Section 4B, where $c$ is an outer automorphism on the Dynkin diagram of even GSpin groups that permutes the last two simple roots.

To explain our results more precisely, let $\boldsymbol{G}_{n}:=\mathbf{G S p i n}_{2 n}$ denote the split even general spin group of semisimple rank $n$ over $F$, and $\mathbf{G} \mathbf{L}_{m}$ the general linear group of semisimple rank $m$. Let $G_{n}$ and $\mathrm{GL}_{m}$ denote the groups of $F$-points of $\boldsymbol{G}_{n}$ and $\mathbf{G L}_{m}$, respectively. Let $R$ and $R_{\mathrm{GL}}$ denote the Grothendieck groups of the category of all admissible representations of finite length of even GSpin and GL groups. Note that $R$ contains two inequivalent representations of the group

MSC2010: primary 20C11; secondary 11F70.

Keywords: strongly positive representations, discrete series representations, Jacquet module. 
$\mathrm{GL}_{n_{1}} \times \mathrm{GL}_{n_{2}} \times \cdots \times \mathrm{GL}_{n_{k}}$ (e.g., $\rho_{1} \otimes \cdots \otimes \rho_{k} \otimes(1 \otimes e)$ and $\rho_{1} \otimes \cdots \otimes \rho_{k} \otimes(1 \otimes c)$, following the notation in Section 2).

Let SP denote the set of all strongly positive representations in $R$, and let $\mathrm{LJ}$ denote the set of pairs (Jord, $\sigma^{\prime}$ ), where

$$
\text { Jord }=\bigcup_{i=1}^{k} \bigcup_{j=1}^{k_{i}}\left\{\left(\rho_{i}, b_{j}^{(i)}\right)\right\}
$$

and $\sigma^{\prime}$ is an irreducible supercuspidal representation in $R$ such that

- $\left\{\rho_{1}, \rho_{2}, \ldots, \rho_{k}\right\} \subset R_{\mathrm{GL}}$ is a (possibly empty) set of mutually nonisomorphic, irreducible and essentially self-dual supercuspidal unitary representations such that $v^{a_{\rho_{i}}} \rho_{i} \rtimes \sigma^{\prime}$ is reducible for $a_{\rho_{i}}>0$ (this defines $a_{\rho_{i}}$ due to the uniqueness of the reducibility point; see Remark 1.2 for more details),

- $k_{i}=\left\lceil a_{\rho_{i}}\right\rceil$, and

- for each $i=1,2, \ldots, k$, the sequence $b_{1}^{(i)}, b_{2}^{(i)}, \ldots, b_{k_{i}}^{(i)}$ of real numbers is such that $-1<b_{1}^{(i)}<b_{2}^{(i)}<\cdots<b_{k_{i}}^{(i)}$ and $a_{\rho_{i}}-b_{j}^{(i)} \in \mathbb{Z}$ for each $j=1, \ldots, k_{i}$.

Remark 1.1. In the set LJ, the condition of "being essentially self-dual" on the representation $\rho_{i}$ for each $i=1, \ldots, k$ is due to certain Weyl group actions on the induced representation $v^{a} \rho_{i} \rtimes \sigma^{\prime}$ (see Corollary 4.6). In the case of even special orthogonal groups, after a minor change to the set LJ, we can construct an SO version of $\mathrm{LJ}$ that corresponds to the set of strongly positive representations of even SO. One minor change would be the condition of "being self-dual" on the corresponding representation $\rho_{i}$ in the case of even special orthogonal groups.

Remark 1.2 [Silberger 1980]. Let $\rho$ and $\sigma$ denote irreducible unitary supercuspidal representations of $\mathrm{GL}_{n}$ and $G_{n}$, respectively. In this paper, we assume that there exists a unique nonnegative real number $a$ such that $\nu^{a} \rho \rtimes \sigma$ reduces. This number $a$ is called the nonnegative rank one reducibility point determined by $\rho$ and $\sigma$.

We construct a bijective mapping as follows (see Theorem 4.16).

Theorem A. There exists a bijective mapping $\Phi$ between SP and LJ. More precisely, consider $\sigma \in \mathrm{SP}$ to be the unique irreducible subrepresentation of the induced representation of the form

$$
\left(\prod_{i=1}^{k} \prod_{j=1}^{k_{i}} \delta\left(\left[v^{a_{\rho_{i}}-k_{i}+j} \rho_{i}, v^{b_{j}^{(i)}} \rho_{i}\right]\right)\right) \rtimes \sigma^{\prime} .
$$

Then, we may define $\Phi(\sigma)$ as

$$
\left(\bigcup_{i=1}^{k} \bigcup_{j=1}^{k_{i}}\left\{\left(\rho_{i}, b_{j}^{(i)}\right)\right\}, \sigma^{\prime}\right) \in \mathrm{LJ} .
$$


To construct $\Phi$, we first classify the special case $D\left(\rho ; \sigma_{\text {cusp }}, c\left(\sigma_{\text {cusp }}\right)\right)$ (see Section $4 \mathrm{~B}$ ), which is the set of strongly positive representations whose supercuspidal supports are the representations $\sigma_{\text {cusp }}, c\left(\sigma_{\text {cusp }}\right)$ of $G_{n}$ and twists of the representation $\rho$ of GL. More precisely, in Theorem 4.9 we construct a bijective mapping between $D\left(\rho ; \sigma_{\text {cusp }}, c\left(\sigma_{\text {cusp }}\right)\right)$ and the set of induced representations of the form

$$
\delta\left(\left[v^{a_{1}} \rho, v^{b_{1}} \rho\right]\right) \times \delta\left(\left[v^{a_{2}} \rho, v^{b_{2}} \rho\right]\right) \times \cdots \times \delta\left(\left[v^{a_{k}} \rho, v^{b_{k}} \rho\right]\right) \rtimes c^{\prime}\left(\sigma_{\text {cusp }}\right),
$$

where $a_{i}=a-k+i, b_{1}<\cdots<b_{k}, k \leq\lceil a\rceil$ and $c^{\prime} \in C:=\{e, c\}$. Here, $a$ is the reducibility point determined by $\rho$ and $\sigma_{\text {cusp }}$, i.e., $v^{s} \rho \rtimes \sigma_{\text {cusp }}$ is reducible if and only if $|s|=a$. We then generalize this result to the set of strongly positive representations. The classification for even GSpin groups needs more work than those for odd GSpin groups since we consider two different representations $\sigma_{\text {cusp }}, c\left(\sigma_{\text {cusp }}\right)$ in $D\left(\rho ; \sigma_{\text {cusp }}, c\left(\sigma_{\text {cusp }}\right)\right)$. (In the odd case, we only need to consider $D\left(\rho ; \sigma_{\text {cusp }}\right)$.)

As an application, our main results give rise to a proof of the equality of $L$ functions through the local Langlands correspondence in the case of GSpin groups [Kim 2015a]. Furthermore, the equality of $L$-functions also has an application in the proof of the generic Arthur packet conjecture in our case. Briefly, the generic Arthur packet conjecture states that if the $L$-packet attached to the Arthur parameter has a generic member, then it is tempered [Shahidi 2011]. This conjecture can be considered a local version of the Generalized Ramanujan Conjecture.

The second purpose of the paper is to explicitly construct Tadić's structure formula for even GSpin groups. Tadić's structure formula studies the Jacquet modules of parabolically induced representations, and it is one of the main tools in the proof of Theorem A. We apply and adapt the ideas from papers of Ban [1999a] and Jantzen [2006] (Tadić's structure formula for even special orthogonal groups) to our case.

The paper is organized as follows. In Section 2, we recall the standard notation and preliminaries. In Section 3, we construct Tadić's structure formula for $G_{n}$ (Theorem 3.4), which gives the explicit structure of the Jacquet modules of the parabolically induced representations of $G_{n}$. In Section 4, we construct the classification of strongly positive representations for $G_{n}$ (Theorem A). In Section 5, we describe embeddings of the general discrete series representations using Casselman's square integrability criterion [Kim 2009] (Theorem 5.1). This embedding of discrete series representations, together with Theorem A, has an interesting application in the proof of the equality of $L$-functions through the local Langlands correspondence for GSpin groups [Kim 2015a].

\section{Notation and preliminaries}

Let $F$ be a nonarchimedean local field of characteristic different from two. For a connected reductive group $\boldsymbol{G}$ defined over $F$, we let $G$ be the group of $F$-points of 
$\boldsymbol{G}$. Let $\boldsymbol{G}_{n}$ be a split even general spin group of semisimple rank $n$ defined over $F$, as defined by Asgari [2002]. A split even GSpin group $\boldsymbol{G}_{n}:=\mathbf{G S p i n}_{2 n}$ is a split reductive linear algebraic group of type $D_{n}$ whose derived group is a double covering of a split special orthogonal group and whose connected component of the Langlands dual group is ${ }^{L} G^{0}=\mathrm{GSO}_{2 n}(\mathbb{C})$. Therefore, the based root datum of $G_{n}$ is the dual based root datum of $\mathrm{GSO}_{2 n}$.

The following proposition describes the structure of GSpin groups as studied by Asgari [2002].

Proposition 2.1. The root datum $\left(X^{*}, R^{*}, X_{*}, R_{*}\right)$ of $G_{n}$ can be described as

$$
\begin{aligned}
& X^{*}=\mathbb{Z} e_{0} \oplus \mathbb{Z} e_{1} \oplus \cdots \oplus \mathbb{Z} e_{n}, \\
& X_{*}=\mathbb{Z} e_{0}^{*} \oplus \mathbb{Z} e_{1}^{*} \oplus \cdots \oplus \mathbb{Z} e_{n}^{*}
\end{aligned}
$$

(there is a standard $\mathbb{Z}$-pairing $\langle$,$\rangle on X^{*} \times X_{*}$ ), with $R^{*}$ and $R_{*}$ generated by

$$
\begin{aligned}
& \Delta^{*}=\left\{\alpha_{1}=e_{1}-e_{2}, \alpha_{2}=e_{2}-e_{3}, \ldots, \alpha_{n-1}=e_{n-1}-e_{n}, \alpha_{n}=e_{n-1}+e_{n}\right\}, \\
& \Delta_{*}=\left\{\alpha_{1}^{\vee}=e_{1}^{*}-e_{2}^{*}, \alpha_{2}^{\vee}=e_{2}^{*}-e_{3}^{*}, \ldots, \alpha_{n-1}^{\vee}=e_{n-1}^{*}-e_{n}^{*}, \alpha_{n}^{\vee}=e_{n-1}^{*}+e_{n}^{*}-e_{0}^{*}\right\} .
\end{aligned}
$$

Let $\boldsymbol{s}=\left(n_{1}, n_{2}, \ldots, n_{k} ; n^{\prime}\right)$ be an ordered partition of $n$. Let $P_{s}=M_{s} N_{s}$ denote the standard parabolic subgroup of $G_{n}$ that corresponds to the partition $s$. The Levi factor $M_{s}$ is isomorphic to $\mathrm{GL}_{n_{1}} \times \mathrm{GL}_{n_{2}} \times \cdots \times \mathrm{GL}_{n_{k}} \times G_{n^{\prime}}$ [Asgari 2002, Theorem 2.7]. When $n^{\prime}=0$ and $n_{k}>0$, we have two nonconjugate standard parabolic subgroups whose Levi subgroups are of the form $\mathrm{GL}_{n_{1}} \times \mathrm{GL}_{n_{2}} \times \cdots \times \mathrm{GL}_{n_{k}}$. In this case, the corresponding set of simple roots contains exactly one of $\alpha_{n-1}, \alpha_{n}$. The corresponding Levi factor is denoted by $M_{\left(n_{1}, \ldots, n_{k} ; 0\right)}=\mathrm{GL}_{n_{1}} \times \mathrm{GL}_{n_{2}} \times \cdots \times \mathrm{GL}_{n_{k}}$, if the corresponding set of simple roots contains $\alpha_{n-1}$; or by $c\left(M_{\left(n_{1}, \ldots, n_{k} ; 0\right)}\right)$ otherwise. Here, we let $c$ be the outer automorphism on the Dynkin diagram of $G_{n}$ that permutes $\alpha_{n-1}$ and $\alpha_{n}$ and fixes other simple roots.

For representations $\rho_{1}, \ldots, \rho_{k}$ of $\mathrm{GL}_{n_{1}}, \ldots, \mathrm{GL}_{n_{k}}$, we let $\rho_{1} \otimes \cdots \otimes \rho_{k} \otimes(1 \otimes e)$ denote a representation of $M_{\left(n_{1}, \ldots, n_{k} ; 0\right)}$, and $\left.\rho_{1} \otimes \cdots \otimes \rho_{k} \otimes(1 \otimes c)\right)$ a representation of $c\left(M_{\left(n_{1}, \ldots, n_{k} ; 0\right)}\right)$. Let $v$ be a character of $\mathrm{GL}_{n}$ defined by $|\operatorname{det}|_{F}$. We denote the induced representation $\operatorname{Ind}_{P}^{G_{n}}\left(\rho_{1} \otimes \cdots \otimes \rho_{k} \otimes \sigma\right)$ by

$$
\rho_{1} \times \cdots \times \rho_{k} \rtimes \sigma
$$

where each $\rho_{i}$ is a representation of some $\mathrm{GL}_{n_{i}}$, and $\sigma$ is a representation of $G_{n}$. We also write $r_{s}(\sigma)$ for the normalized Jacquet module of the representation $\sigma$ with respect to $P_{s}$. In other words, $r_{s}$ is a functor from admissible representations of $G_{n}$ to admissible representations of $M_{s}$. In particular, for a subquotient $\sigma$ of $\rho \rtimes \sigma_{\text {cusp }}$, where $\rho$ is a representation of $\mathrm{GL}_{k}$ and $\sigma_{\text {cusp }}$ is a supercuspidal representation of $G_{n}$, we define $r_{\mathrm{GL}}(\sigma)=r_{(k ; n)}(\sigma)$. 
In the case of GL, for $P^{\prime}=M^{\prime} N^{\prime}$ the standard parabolic subgroup of $\mathrm{GL}_{n}$ with $M^{\prime} \cong \mathrm{GL}_{n_{1}} \times \mathrm{GL}_{n_{2}} \times \cdots \times \mathrm{GL}_{n_{k}}$, we denote the induced representation $\operatorname{Ind}_{P^{\prime}}^{\mathrm{GL}_{n}}\left(\rho_{1} \otimes\right.$ $\left.\cdots \otimes \rho_{k}\right)$ by

$$
\rho_{1} \times \cdots \times \rho_{k}
$$

with each $\rho_{i}$ a representation of some $\mathrm{GL}_{n_{i}}$.

We also follow the notation introduced in [Bernstein and Zelevinsky 1977]. Let $\rho$ be an irreducible unitary supercuspidal representation of some $\mathrm{GL}_{p}$. We define the segment $\Delta:=\left[v^{a} \rho, v^{a+k} \rho\right]=\left\{v^{a} \rho, v^{a+1} \rho, \ldots, v^{a+k} \rho\right\}$, where $a \in \mathbb{R}$ and $k \in \mathbb{Z}_{\geq 0}$. If $a>0$, we call the segment $\Delta$ strongly positive. We note that the induced representation $v^{a+k} \rho \times v^{a+k-1} \rho \times \cdots \times v^{a} \rho$ has a unique irreducible subrepresentation, which we denote by $\delta(\Delta)$. Then $\delta(\Delta)$ is an essentially squareintegrable representation attached to $\Delta$ (Section 3.1 of [Zelevinsky 1980]).

Let us briefly review the Langlands classification for general linear groups. For every irreducible essentially square-integrable representation $\delta$ of some $\mathrm{GL}_{n}$, there exists a unique $e(\delta) \in \mathbb{R}$ such that the representation $v^{-e(\delta)} \delta$ is unitarizable. When $\delta=\delta(\Delta)$, we simply denote $e(\delta(\Delta))$ by $e(\Delta)$. Suppose $\delta_{1}, \delta_{2}, \ldots, \delta_{k}$ are irreducible essentially square-integrable representations of $\mathrm{GL}_{n_{1}}, \mathrm{GL}_{n_{2}}, \ldots, \mathrm{GL}_{n_{k}}$ with $e\left(\delta_{1}\right) \leq e\left(\delta_{2}\right) \leq \cdots \leq e\left(\delta_{k}\right)$. Then, the induced representation $\delta_{1} \times \delta_{2} \times \cdots \times \delta_{k}$ has a unique irreducible subrepresentation, which we denote by $L\left(\delta_{1}, \delta_{2}, \ldots, \delta_{k}\right)$. This irreducible subrepresentation is called the Langlands subrepresentation, and it appears with the multiplicity one in $\delta_{1} \times \delta_{2} \times \cdots \times \delta_{k}$. Every irreducible representation $\rho$ of $\mathrm{GL}_{n}$ is isomorphic to some $L\left(\delta_{1}, \delta_{2}, \ldots, \delta_{k}\right)$. Given $\rho$, the representations $\delta_{1}, \delta_{2}, \ldots, \delta_{k}$ are unique up to a permutation. If $i_{1}, i_{2}, \ldots, i_{k}$ is a permutation of $1,2, \ldots, k$ such that the representations $\delta_{i_{1}} \times \cdots \times \delta_{i_{k}}$ and $\delta_{1} \times \cdots \times \delta_{k}$ are isomorphic, we also write $L\left(\delta_{i_{1}}, \delta_{i_{2}}, \ldots, \delta_{i_{k}}\right)$ for $L\left(\delta_{1}, \delta_{2}, \ldots, \delta_{k}\right)$.

The Grothendieck group of the category of all finite length admissible representations of $G_{n}$ (resp. $\mathrm{GL}_{n}$ ), a free abelian group over the set of all irreducible representations of $G_{n}$ (resp. $\left.\mathrm{GL}_{n}\right)$, is denoted by $R(n)$ (resp. $R_{\mathrm{GL}}(n)$ ). We set

$$
\begin{aligned}
R & =\bigoplus_{n \geq 0} R(n), \\
R_{\mathrm{GL}} & =\bigoplus_{n \geq 0} R_{\mathrm{GL}}(n) .
\end{aligned}
$$

The strongly positive representations of $G_{n}$ are defined as follows.

Definition 2.2 (strongly positive). An irreducible representation $\sigma$ of $G_{n}$ is called strongly positive if for each representation $v^{s_{1}} \rho_{1} \times v^{s_{2}} \rho_{2} \times \cdots \times v^{s_{k}} \rho_{k} \rtimes \sigma_{\text {cusp }}$, where each $\rho_{i}(i=1,2, \ldots, k)$ is an irreducible supercuspidal unitary representation of some $\mathrm{GL}_{n_{i}}, \sigma_{\text {cusp }} \in R$ is an irreducible supercuspidal representation of $G_{n^{\prime}}$ and 
$s_{i} \in \mathbb{R}(i=1,2, \ldots, k)$ is such that

$$
\sigma \hookrightarrow v^{s_{1}} \rho_{1} \times v^{s_{2}} \rho_{2} \times \cdots \times v^{s_{k}} \rho_{k} \rtimes \sigma_{\text {cusp }},
$$

we have $s_{i}>0$ for each $i$.

Finally, the next proposition recalls the properties of discrete series representations studied in [Asgari 2002].

Proposition 2.3. Let $M$ be $\mathrm{GL}_{n_{1}} \times \mathrm{GL}_{n_{2}} \times \cdots \times \mathrm{GL}_{n_{k}} \times G_{n^{\prime}} \subset G_{n}$. Let $\rho_{i}$ be a supercuspidal representation of $\mathrm{GL}_{n_{i}}$ and $\sigma$ a supercuspidal representation of $G_{n^{\prime}}$. Write $\rho_{i}=v^{e\left(\rho_{i}\right)} \rho_{i}^{u}$, where $e\left(\rho_{i}\right) \in \mathbb{R}$ and $\rho_{i}^{u}$ is a unitary supercuspidal representation for $i=1, \ldots, k$. If $\rho_{1} \times \cdots \times \rho_{k} \rtimes \sigma$ has a discrete series subrepresentation, then $\rho_{i}^{u} \cong \tilde{\rho}_{i}^{u} \otimes\left(\omega_{\sigma} \circ \mathrm{det}\right)$ for $i=1, \ldots, k$.

\section{Tadić's structure formula for even GSpin groups}

Tadić's structure formulae for $\mathrm{Sp}_{2 n}, \mathrm{SO}_{2 n+1}, \mathrm{SO}_{2 n}$ and $\mathrm{GSpin}_{2 n+1}$ in [Tadić 1995; Jantzen 2006; Kim 2015b] are based on the geometric lemma (2.11 in [Bernstein and Zelevinsky 1977] or Section 6 in [Casselman 1995]). Briefly, the geometric lemma explicitly calculates the Jacquet modules of induced representations $\left(r_{G, N} \circ\right.$ $i_{G, N}$ in [Bernstein and Zelevinsky 1977]) and it depends on the double coset representative Weyl group elements ( $W^{M, N}$ in [Bernstein and Zelevinsky 1977]) and their actions on the simple roots and representations of Levi subgroups. In this section, we explicitly construct the structure of Jacquet modules of parabolically induced representations of $G_{n}$ (Tadić's structure formula for $G_{n}$, Theorem 3.4) using the geometric lemma. We will adapt and follow the results in [Ban 1999a; Jantzen 2006], i.e., the case of $\mathrm{SO}_{2 n}$. Ban characterizes the double coset representative Weyl group elements ([ $\left.W_{\Omega_{i_{1}}} \backslash W / W_{\Omega_{i_{2}}}\right]$ in [Ban 1999a, Section 5]) and its action on the simple roots in the cases of $D_{n}$-type groups, which include $\mathrm{SO}_{2 n}$ and $G_{n}$. Jantzen [2006] constructs Tadić's structure formula for $\mathrm{SO}_{2 n}$ using Ban's results. Therefore, once we calculate the Weyl group action on the representations in our case, we are ready to adapt Jantzen's calculation [2006] to construct Tadić's structure formula for $G_{n}$.

Let $(p, \epsilon) \in S_{n} \rtimes\{ \pm 1\}^{n}$ be an element in the Weyl group $W_{G_{n}}$ with $\epsilon=$ $\left(\epsilon_{1}, \ldots, \epsilon_{n}\right) \in\{ \pm 1\}^{n}$ such that $\prod_{i=1}^{n} \epsilon_{i}=1$. We can identify $(p, \epsilon) \in W_{G_{n}}$ with $p \cdot \epsilon \in W_{\mathrm{SO}_{2 n}}$ where the action by conjugation of $p$ and $\epsilon \in W_{\mathrm{SO}_{2 n}}$ on the standard maximal torus in $\mathrm{SO}_{2 n}$ can be defined by

$$
\begin{aligned}
& p \cdot \operatorname{diag}\left(x_{1}, \ldots, x_{n}, x_{n}^{-1}, \ldots, x_{1}^{-1}\right)=\operatorname{diag}\left(x_{p^{-1}(1)}, \ldots, x_{p^{-1}(n)}, x_{p^{-1}(n)}^{-1}, \ldots, x_{p^{-1}(1)}^{-1}\right) \\
& \epsilon \cdot \operatorname{diag}\left(x_{1}, \ldots, x_{n}, x_{n}^{-1}, \ldots, x_{1}^{-1}\right)=\operatorname{diag}\left(x_{1}^{\epsilon_{1}}, \ldots, x_{n}^{\epsilon_{n}}, x_{n}^{-\epsilon_{n}}, \ldots, x_{1}^{-\epsilon_{1}}\right) .
\end{aligned}
$$

We can get the action of those on the roots (see also [Hundley and Sayag 2012]). 
Lemma 3.1. Let $e_{0}, e_{1}, \ldots, e_{n}$ and $e_{0}^{\prime}, e_{1}^{\prime}, \ldots, e_{n}^{\prime}$ be the standard bases of the character lattice and the cocharacter lattice of $G_{n}$ as in Proposition 2.1. Let $(p, \epsilon) \in S_{n} \rtimes\{ \pm 1\}^{n}$ be as above.

Then

$$
\begin{aligned}
& (p, \epsilon) \cdot e_{i}= \begin{cases}e_{p(i)} & \text { for } i>0, \epsilon_{i}=1, \\
-e_{p(i)} & \text { for } i>0, \epsilon_{i}=-1, \\
e_{0}+\sum_{\epsilon_{i}=-1} e_{p(i)} & \text { for } i=0,\end{cases} \\
& (p, \epsilon) \cdot e_{i}^{\prime}= \begin{cases}e_{p(i)}^{\prime} & \text { for } i>0, \epsilon_{i}=1, \\
e_{0}^{\prime}-e_{p(i)}^{\prime} & \text { for } i>0, \epsilon_{i}=-1, \\
e_{0}^{\prime} & \text { for } i=0 .\end{cases}
\end{aligned}
$$

Proof. The calculations of $(p, \epsilon) \cdot e_{i}$ for $i>0$ are done in [Ban 1999a, Section 5]. We can also calculate $(p, \epsilon) \cdot e_{i}^{\prime}$ directly from the matrix calculation since $e_{0}^{\prime}, \ldots, e_{n}^{\prime}$ comprise the character lattice of GSO. For $(p, \epsilon) \cdot e_{0}$, we need to use the duality of $e_{i}$ and $e_{i}^{\prime}$.

Let $\Delta^{*}=\left\{\alpha_{1}=e_{1}-e_{2}, \alpha_{2}=e_{2}-e_{3}, \ldots, \alpha_{n-1}=e_{n-1}-e_{n}, \alpha_{n}=e_{n-1}+e_{n}\right\}$ be a simple root for $G_{n}$ as explained in Section 2. From Lemma 3.1, we can calculate the action of $(p, \epsilon)$ on the set of simple roots in $R^{*}$ (see also [Ban 1999a]).

Corollary 3.2. With notation as in Lemma 3.1,

$$
(p, \epsilon) \cdot \alpha_{i}= \begin{cases}\epsilon_{i} e_{p(i)}-\epsilon_{i+1} e_{p(i+1)} & \text { for } 0 \leq i \leq n-1, \\ \epsilon_{n-1} e_{p(n-1)}+\epsilon_{n} e_{p(n)} & \text { for } i=n .\end{cases}
$$

Now we are ready to construct Tadić's structure formula for $G_{n}$. Let $\rho_{i}$ be an irreducible smooth representation of $\mathrm{GL}_{n_{i}}$ for $i=1,2,3,4$. Let $\sigma$ be an irreducible smooth representation in $R$ and let $\omega_{\sigma}$ be the central character of $\sigma$. For any element $c_{1} \in C=\{e, c\}$, we define $\widetilde{\rtimes}$ as follows:

(3-1) $\left(\rho_{1} \otimes \rho_{2} \otimes \rho_{3} \otimes c_{1}\right) \widetilde{\rtimes}\left(\rho_{4} \otimes \sigma\right)=\left(\tilde{\rho}_{1} \otimes\left(\omega_{\sigma} \circ\right.\right.$ det $\left.)\right) \times \rho_{2} \times \rho_{4} \otimes \rho_{3} \rtimes c_{1}(\sigma)$.

One extends $\widetilde{\rtimes}$ to a $\mathbb{Z}$-bilinear mapping

$$
\widetilde{\rtimes}:\left(R_{\mathrm{GL}} \otimes R_{\mathrm{GL}} \otimes R_{\mathrm{GL}} \otimes \mathbb{Z}[C]\right) \times\left(R_{\mathrm{GL}} \otimes R\right) \rightarrow R_{\mathrm{GL}} \otimes R .
$$

We denote by $\boldsymbol{m}$ the linear extension to $R_{\mathrm{GL}} \otimes R_{\mathrm{GL}}$ of parabolic induction from a maximal parabolic subgroup.

Let

$$
\Omega_{k}= \begin{cases}\Delta & \text { if } k=0, \\ \Delta \backslash\left\{\alpha_{k}\right\} & \text { if } k \leq n-2, \\ \Delta \backslash\left\{\alpha_{n-1}, \alpha_{n}\right\} & \text { if } k=n-1, \\ \Delta \backslash\left\{\alpha_{n}\right\} & \text { if } k=n .\end{cases}
$$


and $\bar{\Omega}_{n}=\Delta \backslash\left\{\alpha_{n-1}\right\}=c\left(\Omega_{n}\right)$. We define $\mu^{*}(\sigma)$ as follows. For $0 \leq k \leq n$, write

$$
\begin{aligned}
& r_{M_{\Omega_{k}, G_{n}}}(\sigma)=\sum_{i \in I_{k}} \rho_{i, k} \otimes \sigma_{i, k}, \\
& r_{M_{\bar{\Omega}_{n}, G_{n}}}(\sigma)=\sum_{j \in J} \rho_{j} \otimes(1 \otimes c),
\end{aligned}
$$

the normalized Jacquet modules of $\sigma$ with respect to the standard maximal parabolic subgroups $P_{\Omega_{k}}=M_{\Omega_{k}} N_{\Omega_{k}}$ and $P_{\bar{\Omega}_{n}}=M_{\bar{\Omega}_{n}} N_{\bar{\Omega}_{n}}$, respectively. For such $\sigma$, we can define $\mu^{*}(\sigma) \in R_{\mathrm{GL}} \otimes R$ as

$$
\mu^{*}(\sigma)=\sum_{k=0}^{n} \sum_{i \in I_{k}} \rho_{i, k} \otimes \sigma_{i, k}+\sum_{j \in J} \rho_{j} \otimes(1 \otimes c) .
$$

Using Jacquet modules with respect to the maximal parabolic subgroups of $\mathrm{GL}_{n}$, we can also define

$$
\boldsymbol{m}^{*}(\rho)=\sum_{k=0}^{n} \text { s.s. }\left(r_{k}(\rho)\right) \in R_{\mathrm{GL}} \otimes R_{\mathrm{GL}}
$$

for an irreducible representation $\rho$ of $\mathrm{GL}_{n}$, and then extend $\boldsymbol{m}^{*}$ linearly to the whole of $R_{\mathrm{GL}}$. Here, $r_{k}(\rho)$ denotes the Jacquet module of the representation $\rho$ with respect to the parabolic subgroup whose Levi subgroup is $\mathrm{GL}_{k} \times \mathrm{GL}_{n-k}$, and s.s. denotes so-called semisimplification, i.e., a canonical map from objects of the category of all smooth finite length representations of GL into the Grothendieck group of this category. We define $s: R_{\mathrm{GL}} \otimes R_{\mathrm{GL}} \rightarrow R_{\mathrm{GL}} \otimes R_{\mathrm{GL}}$ by $s(x \otimes y)=y \otimes x$. Let $\mathfrak{M}_{C}^{*}: R_{\mathrm{GL}} \rightarrow R_{\mathrm{GL}} \otimes R_{\mathrm{GL}} \otimes R_{\mathrm{GL}} \otimes \mathbb{Z}[C]$ be the map $\left(1 \otimes \boldsymbol{m}_{C}^{*}\right) \circ \boldsymbol{s} \circ \boldsymbol{m}^{*}$, where

$$
\begin{aligned}
1 \otimes \boldsymbol{m}_{C}^{*}: R_{\mathrm{GL}} \otimes R_{\mathrm{GL}} & \rightarrow R_{\mathrm{GL}} \otimes R_{\mathrm{GL}} \otimes R_{\mathrm{GL}} \otimes \mathbb{Z}[C] \\
\rho_{1} \otimes \rho_{2} & \mapsto \rho_{1} \otimes \boldsymbol{m}^{*}\left(\rho_{2}\right) \otimes c^{n_{1}},
\end{aligned}
$$

for representations $\rho_{1}$ of $\mathrm{GL}_{n_{1}}$ and $\rho_{2}$ of $\mathrm{GL}_{n_{2}}$.

Remark 3.3. In our case of even GSpin groups, we have $c^{n_{1}}$ when we calculate $\mu^{*}$, while we don't have such action in the case of odd GSpin groups. This is due to the differences of the Weyl group actions on the simple roots. In our case, the corresponding Weyl group element acts on $e_{n}$ by $(-1)^{n_{1}}$. Therefore, if $n_{1}$ is odd, we need to permute $\alpha_{n-1}=e_{n-1}-e_{n}$ and $\alpha_{n}=e_{n-1}+e_{n}$. In other words, we have the outer automorphism $c$ on the representation $\sigma$.

The following theorem is called Tadić's structure formula for even GSpin groups.

Theorem 3.4. For $\rho \in R_{\mathrm{GL}}(i)$ and $\sigma \in R(n-i)$, we have

$$
\mu^{*}(\rho \rtimes \sigma)=\mathfrak{M}_{C}^{*}(\rho) \widetilde{\rtimes} \mu^{*}(\sigma) .
$$


Proof. We sketch the proof and explain how we can adapt the approach in [Jantzen 2006] to our case. Let us explicitly calculate the left hand side of the equation in the theorem. Since GSpin $2 n$ is also of $D_{n}$-type, we can apply the double coset representative Weyl group elements, which are studied in [Ban 1999a], to our case. Therefore, we have $\mu^{*}(\rho \rtimes \sigma)$ as in [Jantzen 2006, pp. 811-812]. Now, we calculate the action of the double coset representative Weyl group elements $\left(q_{n}\right.$ in [Jantzen 2006]) on the representations. The actions of $q_{n}$ produce contragredient of $\tau_{s}^{(3)}(d)$ with $\left(\omega_{\theta} \circ\right.$ det $)$, i.e., $\left(\omega_{\theta} \circ\right.$ det $) \tilde{\tau}_{s}^{(3)}(d)$ (see p. 812 for the notation of $\tau_{s}^{(3)}(d)$ and $\left.\theta\right)$. Accordingly, we need to define $\widetilde{\rtimes}$ as (3-1). This forces $\mu^{*}(\rho \rtimes \sigma)$ to be equal to $\mathfrak{M}_{C}^{*}(\rho) \widetilde{\rtimes} \mu^{*}(\sigma)$ after changing index several times as in the proof of [Jantzen 2006, Chapter 4].

\section{Classification of strongly positive representations for even GSpin groups}

We give the classification of strongly positive representations of even GSpin groups in this section. We apply and adapt some proofs from Matić's results [2011] for metaplectic groups to our case. Therefore, we mostly focus on the following cases which are quite different from [Matic 2011]. For example, when the reducibility point is $\frac{1}{2}$, we follow the idea of [Kim 2015b] instead of [Matic 2011]. We also emphasize the difference between the even case and the odd case, and omit the proof if it is similar to the case of either metaplectic groups or odd GSpin groups. For example, in the even case, we need to add $c\left(\sigma_{\text {cusp }}\right)$ when we classify the special case $D\left(\rho ; \sigma_{\text {cusp }}, c\left(\sigma_{\text {cusp }}\right)\right)$ (in the odd case, we classify $D\left(\rho ; \sigma_{\text {cusp }}\right)$ ).

4A. Several lemmas. We recall several lemmas which are essential in this section. Let us first recall the half integer conjecture in the case of GSpin groups. Let $\sigma$ be an irreducible supercuspidal representation of $G_{n^{\prime}}$ and let $\rho$ be an irreducible supercuspidal unitary representation of $\mathrm{GL}_{k}$. The following is a recent result of Mœglin which is called the half integer conjecture for GSpin groups [Mœglin 2014, Theorem 3.1.1]:

Lemma 4.1 [Mœglin 2014]. Let $a \in \mathbb{R}$ be a nonnegative real number such that $\nu^{a} \rho \rtimes \sigma$ reduces. Then, $a \in \frac{1}{2} \mathbb{Z}$.

Remark 4.2. In [Kim 2015b], we classified the strongly positive representations of odd GSpin groups assuming the half integer conjecture. Due to Mœglin's results (Lemma 4.1), we can completely remove the assumption in the odd case.

Remark 4.3. When we further assume that $\sigma$ is a generic representation, Shahidi [1990] proved that $a \in\left\{0, \frac{1}{2}, 1\right\}$.

Let us calculate $\mu^{*}\left(\left(\prod_{j=1}^{n} \delta\left(\left[v^{a_{j}} \rho_{j}, v^{b_{j}} \rho_{j}\right]\right)\right) \rtimes \sigma\right)$, where each $\rho_{j}$ is an irreducible supercuspidal representation of $\mathrm{GL}_{k}$, the real numbers $a_{j}$ and $b_{j}$ are such 
that $b_{j}-a_{j} \in \mathbb{Z}_{\geq 0}$ for each $j=1, \ldots, n$ and $\sigma$ is an irreducible supercuspidal representation of finite length of $G_{n^{\prime}}$.

Example 4.4. Let us first calculate the case when $n=1$. Since $\sigma$ is supercuspidal, $\mu^{*}(\sigma)=1 \otimes \sigma$. Following the definition of $\mathfrak{M}_{C}^{*}$, we have

$$
\begin{aligned}
& \mathfrak{M}_{C}^{*}\left(\delta\left(\left[v^{a} \rho_{1}, v^{b} \rho_{1}\right]\right)\right) \\
& =\sum_{i=a-1}^{b} \sum_{j=i}^{b} \delta\left(\left[v^{a} \rho_{1}, v^{i} \rho_{1}\right]\right) \otimes \delta\left(\left[v^{j+1} \rho_{1}, v^{b} \rho_{1}\right]\right) \otimes \delta\left(\left[v^{i+1} \rho_{1}, v^{j} \rho_{1}\right]\right) \otimes c^{k(i-a+1)} .
\end{aligned}
$$

Therefore,

$$
\begin{aligned}
\mu^{*}\left(\delta\left(\left[v^{a} \rho_{1}, v^{b} \rho_{1}\right]\right) \rtimes \sigma\right) & =\sum_{i=a-1}^{b} \sum_{j=i}^{b} \delta\left(\left[v^{-i} \tilde{\rho}_{1} \otimes\left(\omega_{\sigma} \circ \operatorname{det}\right), v^{-a} \tilde{\rho}_{1} \otimes\left(\omega_{\sigma} \circ \text { det }\right)\right]\right) \\
& \times \delta\left(\left[v^{j+1} \rho_{1}, v^{b} \rho_{1}\right]\right) \otimes \delta\left(\left[v^{i+1} \rho_{1}, v^{j} \rho_{1}\right]\right) \rtimes c^{k(i-a+1)}(\sigma) .
\end{aligned}
$$

We omit $\delta\left(\left[v^{x} \rho_{1}, v^{y} \rho_{1}\right]\right)$ if $x>y$. Then, to calculate

$$
\mu^{*}\left(\left(\prod_{j=1}^{n} \delta\left(\left[v^{a_{j}} \rho_{j}, v^{b_{j}} \rho_{j}\right]\right)\right) \rtimes \sigma\right),
$$

we use (1.3) of [Tadić 1998]:

$m^{*}\left(\prod_{j=1}^{n} \delta\left(\left[v^{a_{j}} \rho_{j}, v^{b_{j}} \rho_{j}\right]\right)\right)=\prod_{j=1}^{n}\left(\sum_{i_{j}=a_{j}-1}^{b_{j}} \delta\left(\left[v^{i_{j}+1} \rho_{j}, v^{b_{j}} \rho_{j}\right]\right) \otimes \delta\left(\left[v^{a_{j}} \rho_{j}, v^{i_{j}} \rho_{j}\right]\right)\right)$.

The Weyl group elements are essential objects when we define the intertwining operators between two induced representations [Shahidi 2010, Chapter 4]. We recall the action of the Weyl group elements on the induced representations. Let $M_{\theta}$ be a Levi subgroup isomorphic to $\mathrm{GL}_{k} \times G_{n-k}$ for $\theta=\Delta \backslash \alpha_{k}$. There is a unique Weyl group element $w_{0}$ such that $w_{0}\left(\alpha_{k}\right)<0$ and $w_{0}(\theta) \subset \Delta$.

Lemma 4.5. Let $\rho$ and $\sigma$ be irreducible supercuspidal representations of $\mathrm{GL}_{k}$ and $G_{n-k}$, respectively. Then

$$
(\rho \otimes \sigma)^{w_{0}}=\left(\tilde{\rho} \otimes\left(\omega_{\sigma} \circ \text { det }\right)\right) \otimes c^{k}(\sigma),
$$

where $\omega_{\sigma}$ is the central character of $\sigma$.

Proof. Since $w_{0}\left(\alpha_{k}\right)<0$ and $w_{0}(\theta) \subset \Delta$, we can explicitly calculate its action on the simple roots. Let us identify $w_{0}$ as $(p, \epsilon) \in S_{n} \rtimes\{ \pm 1\}^{n}$ with $\epsilon=\left(\epsilon_{1}, \ldots, \epsilon_{n}\right) \in\{ \pm 1\}^{n}$ such that $\prod_{i=1}^{n} \epsilon_{i}=1$. Then 
$p(i)=\left\{\begin{array}{ll}k+1-i & \text { for } 1 \leq i \leq k, \\ i & \text { for } k+1 \leq i \leq n,\end{array} \quad\right.$ and $\quad \epsilon_{i}= \begin{cases}-1 & \text { for } 1 \leq i \leq k, \\ 1 & \text { for } k+1 \leq i \leq n \\ (-1)^{k} & \text { for } i=n,\end{cases}$

for $k \neq n-1, n$, and

$$
p(i)=n+1-i \quad \text { and } \quad \epsilon_{i}= \begin{cases}(-1)^{k-1} & \text { for } i=1, \\ -1 & \text { for } 2 \leq i \leq n-1, \\ (-1)^{k+n-1} & \text { for } i=n\end{cases}
$$

for $k=n-1, n$. Using this identification, the lemma follows from Lemma 3.1.

Corollary 4.6. Let $\rho$ and $\sigma$ be as in Lemma 4.5. Then $\rho \rtimes \sigma$ and $\left(\tilde{\rho} \otimes\left(\omega_{\sigma} \circ \mathrm{det}\right)\right) \rtimes$ $c^{k}(\sigma)$ are associate. Therefore, Lemma 5.4 (iii) of [Bernstein et al. 1986] implies that the sets of irreducible composition factors of $\rho \rtimes \sigma$ and $\left(\tilde{\rho} \otimes\left(\omega_{\sigma} \circ \mathrm{det}\right)\right) \rtimes c^{k}(\sigma)$ are the same. Furthermore, if we assume that $\rho \rtimes \sigma$ is irreducible, then $\rho \rtimes \sigma \cong$ $\left(\tilde{\rho} \otimes\left(\omega_{\sigma} \circ \mathrm{det}\right)\right) \rtimes c^{k}(\sigma)$.

Now we show that strongly positive representations can be embedded into parabolically induced representations of special type. More precisely, we consider parabolically induced representations of the form

$$
\delta\left(\Delta_{1}\right) \times \delta\left(\Delta_{2}\right) \times \cdots \times \delta\left(\Delta_{k}\right) \rtimes c^{\prime}\left(\sigma_{\text {cusp }}\right),
$$

where $\Delta_{1}, \Delta_{2}, \ldots, \Delta_{k}$ is a sequence of strongly positive segments satisfying $0<e\left(\Delta_{1}\right) \leq e\left(\Delta_{2}\right) \leq \cdots \leq e\left(\Delta_{k}\right)$ (we allow $k=0$ here), $\sigma_{\text {cusp }}$ is an irreducible supercuspidal representation of $G_{n^{\prime}}$ and $c^{\prime} \in C$. Note that the idea of such embeddings of representations was initiated in [Muić 2006] and further refined in [Hanzer and Muić 2008].

Lemma 4.7. Let $\Delta_{1}, \ldots, \Delta_{k}$ and $\sigma_{\text {cusp }}$ be as above. Then the induced representation $\delta\left(\Delta_{1}\right) \times \delta\left(\Delta_{2}\right) \times \cdots \times \delta\left(\Delta_{k}\right) \rtimes c^{\prime}\left(\sigma_{\text {cusp }}\right)$ has a unique irreducible subrepresentation, which we denote by $\delta\left(\Delta_{1}, \ldots, \Delta_{k} ; c^{\prime}\left(\sigma_{\text {cusp }}\right)\right)$.

Proof. We briefly explain the main ideas of the proof and how we adapt the proof from [Matić 2011] to the case of even GSpin groups. The case $k=0$ is clear. Let $j_{1}<j_{2}<\cdots<j_{s}$ be the positive integers such that

$e\left(\Delta_{1}\right)=\cdots=e\left(\Delta_{j_{1}}\right)<e\left(\Delta_{j_{1}+1}\right)=\cdots=e\left(\Delta_{j_{2}}\right)<\cdots<e\left(\Delta_{j_{s}+1}\right)=\cdots=e\left(\Delta_{k}\right)$.

Then Theorem 3.4 implies that an irreducible representation

$$
\delta\left(\Delta_{1}\right) \times \cdots \times \delta\left(\Delta_{j_{1}}\right) \otimes \delta\left(\Delta_{j_{1}+1}\right) \times \cdots \times \delta\left(\Delta_{j_{2}}\right) \otimes \cdots \otimes c^{\prime}\left(\sigma_{\text {cusp }}\right)
$$

appears with multiplicity one in the Jacquet module of $\delta\left(\Delta_{1}\right) \times \delta\left(\Delta_{2}\right) \times \cdots \times$ $\delta\left(\Delta_{k}\right) \rtimes c^{\prime}\left(\sigma_{\text {cusp }}\right)$ with respect to the appropriate parabolic subgroup. Therefore, since this irreducible representation is contained in the Jacquet module of any 
subrepresentation of $\delta\left(\Delta_{1}\right) \times \delta\left(\Delta_{2}\right) \times \cdots \times \delta\left(\Delta_{k}\right) \rtimes c^{\prime}\left(\sigma_{\text {cusp }}\right)$ with respect to the appropriate parabolic subgroup, it follows that the induced representation $\delta\left(\Delta_{1}\right) \times$ $\delta\left(\Delta_{2}\right) \times \cdots \times \delta\left(\Delta_{k}\right) \rtimes c^{\prime}\left(\sigma_{\text {cusp }}\right)$ has a unique irreducible subrepresentation.

Now, as in the odd case [Kim 2015b], we can show that strongly positive representations can be embedded into induced representations of the form (4-1).

Lemma 4.8. Let $\sigma \in R$ denote a strongly positive representation. Then $\sigma$ can be embedded into certain induced representations of the form (4-1).

\section{B. Classification of strongly positive representations: the $D\left(\rho ; \sigma_{\mathrm{cusp}}, c\left(\sigma_{\mathrm{cusp}}\right)\right)$}

case. Let $\rho$ be an essentially self-dual irreducible supercuspidal representation of $\mathrm{GL}_{n_{\rho}}$ and $\sigma_{\text {cusp }}$ an irreducible supercuspidal representation of $G_{n^{\prime}}$. Also, let $D\left(\rho ; \sigma_{\text {cusp }}, c\left(\sigma_{\text {cusp }}\right)\right)$ be the set of strongly positive representations whose supercuspidal supports are the representations $\sigma_{\text {cusp }}, c\left(\sigma_{\text {cusp }}\right)$ and twists of the representation $\rho$. We assume that there exists a unique nonnegative real number $a$ such that $\nu^{a} \rho \rtimes \sigma_{\text {cusp }}$ reduces [Silberger 1980]. The half integer conjecture for GSpin groups (Lemma 4.1) implies that $a \in \frac{1}{2} \mathbb{Z}$.

In this section, we construct the classification of strongly positive representations in $D\left(\rho ; \sigma_{\text {cusp }}, c\left(\sigma_{\text {cusp }}\right)\right)$. Lemma 4.8 implies that there exists a mapping from the set of strongly positive representations of $G_{n}$ into the set of induced representations of the form (4-1). We first refine the image of this map when we restrict the map to $D\left(\rho ; \sigma_{\text {cusp }}, c\left(\sigma_{\text {cusp }}\right)\right)$.

Theorem 4.9. Suppose that $\sigma$ is an irreducible strongly positive representation in $D\left(\rho ; \sigma_{\text {cusp }}, c\left(\sigma_{\text {cusp }}\right)\right)$, taken as the unique irreducible subrepresentation of an induced representation of the form (4-1). Write $\Delta_{i}=\left[v^{a_{i}} \rho, v^{b_{i}} \rho\right]$ for $i=1, \ldots, k$. Then $a_{i}=a-k+i$ for each $i, b_{1}<\cdots<b_{k}$ and $k \leq\lceil a\rceil$.

Proof. We consider the case when $a=\frac{1}{2}$. We first show, by induction on $k$, that $a_{i}=a$ for each $i=1, \ldots, k$ and that $b_{1} \leq \cdots \leq b_{k}$ when $a=\frac{1}{2}$.

For the case $k=1$, note that if $a_{i} \neq a$, then

$$
v^{a_{i}} \rho \rtimes \sigma_{\text {cusp }} \cong\left(v^{-a_{i}} \tilde{\rho} \otimes\left(\omega_{\sigma_{\text {cusp }}} \circ \text { det }\right)\right) \rtimes c^{n_{\rho}}\left(\sigma_{\text {cusp }}\right) \cong v^{-a_{i}} \rho \rtimes \sigma_{\text {cusp }} .
$$

Therefore, we have the embedding

$$
\sigma \hookrightarrow v^{b_{1}} \rho \times \cdots \times v^{a_{1}+1} \rho \times v^{-a_{1}} \rho \rtimes \sigma_{\text {cusp }}
$$

which contradicts the strong positivity of $\sigma$.

Now suppose the theorem holds for all $m \in \mathbb{Z}$ such that $0 \leq m<k$. We prove it for $k$. As in the case when $k=1$, we show $a_{k}=a$. We know that $\sigma$ embeds in $\delta\left(\Delta_{1}\right) \rtimes \delta\left(\Delta_{2}, \ldots, \Delta_{k} ; \sigma_{\text {cusp }}\right)$, since $\sigma$ is the unique irreducible subrepresentation of $\delta\left(\Delta_{1}\right) \times \cdots \delta\left(\Delta_{k}\right) \rtimes \sigma_{\text {cusp. }}$. This implies that each $a_{i}=a$ for $2 \leq i \leq k$ and $b_{2} \leq \cdots \leq b_{k}$. It remains to show that $a_{1}=\frac{1}{2}$ and $b_{1} \leq b_{2}$. 
Suppose that $a_{1} \notin \frac{1}{2}+\mathbb{Z}$. Then $v^{a_{1}} \rho \times \delta\left(\Delta_{i}\right)$ is irreducible for $i \geq 2$. Therefore, we have the embedding

$$
\begin{aligned}
\sigma \hookrightarrow \delta\left(\left[v^{a_{1}+1} \rho, v^{b_{1}} \rho\right]\right) \times v^{a_{1}} \rho \times \delta\left(\Delta_{2}\right) \times \cdots \times \delta\left(\Delta_{k}\right) \rtimes \sigma_{\text {cusp }} \\
\cong \delta\left(\left[v^{a_{1}+1} \rho, v^{b_{1}} \rho\right]\right) \times \delta\left(\Delta_{2}\right) \times \cdots \times \delta\left(\Delta_{k}\right) \times v^{a_{1}} \rho \rtimes \sigma_{\text {cusp }} \\
\cong \delta\left(\left[v^{a_{1}+1} \rho, v^{b_{1}} \rho\right]\right) \times \delta\left(\Delta_{2}\right) \times \cdots \times \delta\left(\Delta_{k}\right) \times v^{-a_{1}} \rho \rtimes \sigma_{\text {cusp }}
\end{aligned}
$$

which contradicts the strong positivity of $\sigma$. Therefore, $a_{1} \in \frac{1}{2}+\mathbb{Z}$. If $a_{1} \neq \frac{1}{2}$, then $a_{1} \geq \frac{3}{2}$. This implies that $\delta\left(\Delta_{1}\right) \times \delta\left(\Delta_{i}\right)$ is irreducible for $i \geq 2$ since $b_{1} \leq b_{i}$. Therefore, we have the embedding

$$
\begin{aligned}
\sigma \hookrightarrow \delta\left(\Delta_{2}\right) \times \cdots \times & \delta\left(\Delta_{k}\right) \times \delta\left(\Delta_{1}\right) \rtimes \sigma_{\text {cusp }} \\
\cong \delta\left(\Delta_{2}\right) & \times \cdots \times \delta\left(\Delta_{k}\right) \times \delta\left(\left[v^{a_{1}+1} \rho, v^{b_{1}} \rho\right]\right) \times v^{a_{1}} \rho \rtimes \sigma_{\text {cusp }} \\
& \cong \delta\left(\Delta_{2}\right) \times \cdots \times \delta\left(\Delta_{k}\right) \times \delta\left(\left[v^{a_{1}+1} \rho, v^{b_{1}} \rho\right]\right) \times v^{-a_{1}} \rho \rtimes \sigma_{\text {cusp }}
\end{aligned}
$$

which again contradicts the strong positivity of $\sigma$. Thus, $a_{1}=\frac{1}{2}$. Also, $b_{1} \leq b_{2}$ follows from $e\left(\Delta_{1}\right) \leq e\left(\Delta_{2}\right)$. The following lemma finishes the proof of the theorem in the case when $a=\frac{1}{2}$.

Lemma 4.10. The unique irreducible subrepresentation of

$$
\delta\left(\left[v^{1 / 2} \rho, v^{b_{1}^{\prime}} \rho\right]\right) \times \delta\left(\left[v^{1 / 2} \rho, v^{b_{2}^{\prime}} \rho\right]\right) \times \cdots \times \delta\left(\left[v^{1 / 2} \rho, v^{b_{k}^{\prime}} \rho\right]\right) \rtimes \sigma_{\text {cusp }},
$$

denoted $\sigma_{\left(b_{1}^{\prime}, \ldots, b_{k}^{\prime} ; 1 / 2\right)}^{*}$, is not strongly positive when $k \geq 2$.

Proof. We first show the case when $k=2$. The embedding of $v^{1 / 2} \rho \rtimes \delta\left(v^{1 / 2} \rho, \sigma_{\text {cusp }}\right)$ into $v^{1 / 2} \rho \times v^{1 / 2} \rho \rtimes \sigma_{\text {cusp }}$ implies the embedding

$$
\sigma_{(1 / 2,1 / 2 ; 1 / 2)}^{*} \hookrightarrow v^{1 / 2} \rho \rtimes \delta\left(v^{1 / 2} \rho, \sigma_{\text {cusp }}\right) .
$$

Using Lemma 3.8 (b) as well as Remark 3.2 of [Tadic 1998], we can show that $\delta\left(\left[v^{-1 / 2} \rho, v^{1 / 2} \rho\right]\right) \rtimes \sigma_{\text {cusp }}$ is a direct sum of two irreducible nonisomorphic representations, say $\tau_{1}$ and $\tau_{2}$, in the same way as in the proof of Sublemma 5.8 of [Kim 2015b]. From Frobenius reciprocity and

$$
\begin{aligned}
r_{\mathrm{GL}}\left(\delta\left(\left[v^{-1 / 2} \rho, v^{1 / 2} \rho\right]\right)\right. & \left.\rtimes \sigma_{\text {cusp }}\right)= \\
& 2 \delta\left(\left[v^{-1 / 2} \rho, v^{1 / 2} \rho\right]\right) \otimes \sigma_{\text {cusp }}+v^{1 / 2} \rho \times v^{1 / 2} \rho \otimes c^{n_{\rho}}\left(\sigma_{\text {cusp }}\right),
\end{aligned}
$$

it follows that $r_{\mathrm{GL}}\left(\tau_{1}\right)=\delta\left(\left[v^{-1 / 2} \rho, v^{1 / 2} \rho\right]\right) \otimes \sigma_{\text {cusp }}+v^{1 / 2} \rho \times v^{1 / 2} \rho \otimes c^{n_{\rho}}\left(\sigma_{\text {cusp }}\right)$ and $r_{\mathrm{GL}}\left(\tau_{2}\right)=\delta\left(\left[v^{-1 / 2} \rho, v^{1 / 2} \rho\right]\right) \otimes \sigma_{\text {cusp }}$. We also obtain that $\sigma_{(1 / 2,1 / 2 ; 1 / 2)}^{*} \cong \tau_{1}$, which is a subrepresentation of $\delta\left(\left[v^{-1 / 2} \rho, v^{1 / 2} \rho\right]\right) \rtimes \sigma_{\text {cusp }}$, in the same way as in the proof of Sublemma 5.9 of [Kim 2015b]. Therefore, we have the embedding

$$
\sigma_{(1 / 2,1 / 2 ; 1 / 2)}^{*} \hookrightarrow \delta\left(\left[v^{-1 / 2} \rho, v^{1 / 2} \rho\right]\right) \rtimes \sigma_{\text {cusp }} \hookrightarrow v^{1 / 2} \rho \times v^{-1 / 2} \rho \rtimes \sigma_{\text {cusp }} .
$$


We conclude that $\sigma_{(1 / 2,1 / 2 ; 1 / 2)}^{*}$ is not strongly positive.

Next we consider the case $k \geq 2$. Suppose that $\sigma_{\left(b_{1}^{\prime}, \ldots, b_{k}^{\prime} ; 1 / 2\right)}^{*}$ is strongly positive. Since each representation $v^{1 / 2} \rho \times \delta\left(\left[v^{1 / 2} \rho, v^{b_{i}^{\prime}} \rho\right]\right)$ is irreducible for all $i=1, \ldots, k$, we have an embedding of $\sigma_{\left(b_{1}^{\prime}, \ldots, b_{k}^{\prime} ; 1 / 2\right)}^{*}$ into

$$
\begin{aligned}
& \delta\left(\left[v^{3 / 2} \rho, v^{b_{1}^{\prime}} \rho\right]\right) \times \delta\left(\left[v^{3 / 2} \rho, v^{b_{2}^{\prime}} \rho\right]\right) \times \\
& \cdots \times \delta\left(\left[v^{3 / 2} \rho, v^{b_{k}^{\prime}} \rho\right]\right) \times v^{1 / 2} \rho \times \cdots \times v^{1 / 2} \rho \rtimes \sigma_{\text {cusp }} .
\end{aligned}
$$

Furthermore, since we know $\sigma_{(1 / 2,1 / 2 ; 1 / 2)}^{*}$ is the unique irreducible subrepresentation of $v^{1 / 2} \rho \times v^{1 / 2} \rho \rtimes \sigma_{\text {cusp }}$, we have the embedding

$$
\sigma_{\left(b_{1}^{\prime}, \ldots, b_{k}^{\prime} ; 1 / 2\right)}^{*} \hookrightarrow \delta\left(\left[v^{3 / 2} \rho, v^{b_{1}^{\prime}} \rho\right]\right) \times \cdots \times v^{1 / 2} \rho \rtimes \sigma_{(1 / 2,1 / 2 ; 1 / 2)}^{*} .
$$

This contradicts the strong positivity of $\sigma_{(1 / 2,1 / 2 ; 1 / 2)}^{*}$.

Returning to the proof of Theorem 4.9, it remains to prove the case when $a \neq \frac{1}{2}$. This case is similar to the proof in [Matić 2011] and we skip the proof here.

We also show that the map from $D\left(\rho ; \sigma_{\text {cusp }}, c\left(\sigma_{\text {cusp }}\right)\right)$ to the set of induced representations of the form (4-1) is well defined in the following theorem.

Theorem 4.11. Suppose that $\sigma$ is an irreducible strongly positive representation in $D\left(\rho ; \sigma_{\text {cusp }}, c\left(\sigma_{\text {cusp }}\right)\right)$. Then there exist a unique set of strongly positive segments $\Delta_{1}, \Delta_{2}, \ldots, \Delta_{k}$ with $0<e\left(\Delta_{1}\right) \leq e\left(\Delta_{2}\right) \leq \cdots \leq e\left(\Delta_{k}\right)$, and a unique irreducible supercuspidal representation $\sigma^{\prime} \in R$ such that $\sigma \simeq \delta\left(\Delta_{1}, \Delta_{2}, \ldots, \Delta_{k} ; \sigma^{\prime}\right)$.

Proof. We first show the uniqueness of the partial supercuspidal support $\sigma^{\prime}$. Suppose that there are two sets of strongly positive segments and representations in $R,\left\{\Delta_{1}, \Delta_{2}, \ldots, \Delta_{k}, \sigma_{\text {cusp }}\right\}$ and $\left\{\Delta_{1}^{\prime}, \Delta_{2}^{\prime}, \ldots, \Delta_{l}^{\prime}, c\left(\sigma_{\text {cusp }}\right)\right\}$, which satisfy the conditions in Theorem 4.9. Then we have the two embeddings

$$
\begin{aligned}
& \sigma \hookrightarrow\left(\prod_{i=1}^{k} \delta\left(\Delta_{i}\right)\right) \rtimes \sigma_{\text {cusp }}, \\
& \sigma \hookrightarrow\left(\prod_{j=1}^{l} \delta\left(\Delta_{j}^{\prime}\right)\right) \rtimes c\left(\sigma_{\text {cusp }}\right) .
\end{aligned}
$$

These embeddings imply that

$$
\left(\prod_{j=1}^{l} \delta\left(\Delta_{j}^{\prime}\right)\right) \otimes c\left(\sigma_{\text {cusp }}\right) \leq r_{\mathrm{GL}}(\sigma) \leq r_{\mathrm{GL}}\left(\left(\prod_{i=1}^{k} \delta\left(\Delta_{i}\right)\right) \rtimes \sigma_{\text {cusp }}\right) .
$$

However, Theorem 3.4 implies that $r_{\mathrm{GL}}\left(\left(\prod_{i=1}^{k} \delta\left(\Delta_{i}\right)\right) \rtimes \sigma_{\text {cusp }}\right)$ can contain the support $c\left(\sigma_{\text {cusp }}\right)$ only if the corresponding GL part, $\prod_{j=1}^{l} \delta\left(\Delta_{j}^{\prime}\right)$, has negative exponent. This is a contradiction since each $\Delta_{i}^{\prime}$ is strongly positive for all $i$. 
It remains to show the uniqueness of strongly positive segments. The situation becomes similar to the odd case [Kim 2015b] since we show the uniqueness of $\sigma^{\prime}$. Therefore, we can apply the idea of [Matić 2011] (for $a \neq \frac{1}{2}$ ) and [Kim 2015b] (for $a=\frac{1}{2}$ ) to complete the proof, which we omit here.

In Theorem 4.9 and Theorem 4.11, we construct an injective mapping from $D\left(\rho ; \sigma_{\text {cusp }}, c\left(\sigma_{\text {cusp }}\right)\right)$ into the set of induced representations of the form

$$
\delta\left(\left[v^{a-k+1} \rho, v^{b_{1}^{\prime}} \rho\right]\right) \times \delta\left(\left[v^{a-k+2} \rho, v^{b_{2}^{\prime}} \rho\right]\right) \times \cdots \times \delta\left(\left[v^{a} \rho, v^{b_{k}^{\prime}} \rho\right]\right) \rtimes c^{\prime}\left(\sigma_{\text {cusp }}\right)
$$

((4-1) with refinement on the unitary exponents as in Theorem 4.9). In other words, if we let $\operatorname{Jord}_{(\rho, a)}^{c^{\prime}}$ be the set of $\left(c^{\prime} ; b_{1}, b_{2}, \ldots, b_{k_{\rho}}\right)$, where $c^{\prime} \in C$ and $b_{i} \in \mathbb{R}$ are such that $b_{i}-a+k_{\rho}-i \in \mathbb{Z}_{\geq 0}$ for $i=1, \ldots, k_{\rho}$ and $-1<b_{1}<b_{2}<\cdots<b_{k_{\rho}}$, we construct the injective mapping

$$
D\left(\rho ; \sigma_{\text {cusp }}, c\left(\sigma_{\text {cusp }}\right)\right) \hookrightarrow \operatorname{Jord}_{(\rho, a)}^{e} \cup \operatorname{Jord}_{(\rho, a)}^{c} .
$$

It remains to show that this injective mapping is also surjective. For an element $\left(c^{\prime} ; b_{1}, b_{2}, \ldots, b_{k_{\rho}}\right) \in \operatorname{Jord}_{(\rho, a)}^{e} \cup \operatorname{Jord}_{(\rho, a)}^{c}$, let $\sigma_{\left(c^{\prime} ; b_{1}, \ldots, b_{k_{\rho}} ; a\right)}$ be a unique irreducible subrepresentation of

$$
\delta\left(\left[v^{a-k_{\rho}+1} \rho, v^{b_{1}} \rho\right]\right) \times \delta\left(\left[v^{a-k_{\rho}+2} \rho, v^{b_{2}} \rho\right]\right) \times \cdots \times \delta\left(\left[v^{a} \rho, v^{b_{k_{\rho}}} \rho\right]\right) \rtimes c^{\prime}\left(\sigma_{\text {cusp }}\right) .
$$

To show the surjectivity, we apply the induction argument in [Matic 2011] to show that the above subrepresentation $\sigma_{\left(c^{\prime} ; b_{1}, \ldots, b_{k_{\rho}} ; a\right)}$ is strongly positive. We don't repeat the argument here.

Theorem 4.12. The representation $\sigma_{\left(c^{\prime} ; b_{1}, \ldots, b_{k_{\rho}} ; a\right)}$ is strongly positive.

Remark 4.13. In the case of odd GSpin groups, we classify the special case $D\left(\rho ; \sigma_{\text {cusp }}\right)$ in [Kim $\left.2015 \mathrm{~b}\right]$. In the even case, we need to consider $c\left(\sigma_{\text {cusp }}\right)$ as a support in $D\left(\rho ; \sigma_{\text {cusp }}, c\left(\sigma_{\text {cusp }}\right)\right)$ as well, since the action of certain Weyl group elements makes $\sigma$ into $c(\sigma)$ (e.g., Lemma 4.5).

4C. Classification of strongly positive representations. Let $\rho_{i}$ be an essentially self-dual irreducible supercuspidal representation of $\mathrm{GL}_{n_{\rho_{i}}}$ for $i=1, \ldots, k$, and $\sigma_{\text {cusp }}$ an irreducible supercuspidal representation of $G_{n^{\prime}}$. We consider the set $D\left(\rho_{1}, \rho_{2}, \ldots, \rho_{k} ; \sigma_{\text {cusp }}, c\left(\sigma_{\text {cusp }}\right)\right)$ of strongly positive representations whose supercuspidal supports are the representations $\sigma_{\text {cusp }}, c\left(\sigma_{\text {cusp }}\right)$ and twists of the representations $\rho_{1}, \ldots, \rho_{k}$. We assume that there exists a unique nonnegative real number $a_{\rho_{i}}$ such that $v^{a_{\rho_{i}}} \rho_{i} \rtimes \sigma_{\text {cusp }}$ reduces for each $i$ [Silberger 1980]. The half integer conjecture for GSpin groups (i.e., Lemma 4.1) implies that each $a_{\rho_{i}} \in \frac{1}{2} \mathbb{Z}$. 
Theorem 4.14. Let $\sigma$ be a strongly positive representation in $D\left(\rho_{1}, \rho_{2}, \ldots, \rho_{k}\right.$; $\left.\sigma_{\text {cusp }}, c\left(\sigma_{\text {cusp }}\right)\right)$. Then $\sigma$ can be considered to be the unique irreducible subrepresentation of the induced representation

$$
\left(\prod_{i=1}^{k} \prod_{j=1}^{k_{i}} \delta\left(\left[v^{a_{\rho_{i}}-k_{i}+j} \rho_{i}, v^{b_{j}^{(i)}} \rho_{i}\right]\right)\right) \rtimes c^{\prime}\left(\sigma_{\text {cusp }}\right),
$$

where $c^{\prime} \in C=\{e, c\}$ and for $i=1, \ldots, k$ and $j=1, \ldots, k_{i}$, each $k_{i} \in \mathbb{Z}_{\geq 0}$ satisfies $k_{i} \leq\left\lceil a_{\rho_{i}}\right\rceil$ and each $b_{j}^{(i)}>0$ is such that $b_{j}^{(i)}-a_{\rho_{i}} \in \mathbb{Z}_{\geq 0}$. Also, $b_{j}^{(i)}<b_{j+1}^{(i)}$ for $1 \leq j \leq k_{i}-1$.

Proof. The proof is exactly the same as the odd case, and so is omitted.

We also show that the map from $D\left(\rho_{1}, \rho_{2}, \ldots, \rho_{k} ; \sigma_{\text {cusp }}, c\left(\sigma_{\text {cusp }}\right)\right)$ to the set of induced representations of the form (4-4) is well defined in the following theorem.

Theorem 4.15. Suppose that the representation $\sigma$ is the unique irreducible subrepresentations of both representations

$$
\begin{aligned}
& \left(\prod_{i=1}^{k} \prod_{j=1}^{k_{i}} \delta\left(\left[v^{a_{\rho_{i}}-k_{i}+j} \rho_{i}, v^{b_{j}^{(i)}} \rho_{i}\right]\right)\right) \rtimes \sigma_{\text {cusp }}, \\
& \left(\prod_{i=1}^{k^{\prime}} \prod_{j=1}^{k_{i}^{\prime}} \delta\left(\left[v^{a \rho_{i}^{\prime}-k_{i}^{\prime}+j} \rho_{i}^{\prime}, v^{c_{j}^{(i)}} \rho_{i}^{\prime}\right]\right)\right) \rtimes c^{\prime}\left(\sigma_{\text {cusp }}\right)
\end{aligned}
$$

as in Theorem 4.14. Then $k=k^{\prime}, \sigma_{\text {cusp }} \cong c^{\prime}\left(\sigma_{\text {cusp }}\right)$ and

$$
\left\{\prod_{j=1}^{k_{i}} \delta\left(\left[v^{a_{\rho_{i}}-k_{i}+j} \rho_{i}, v^{b_{j}^{(i)}} \rho_{i}\right]\right) \mid i=1, \ldots, k\right\}
$$

is a permutation of

$$
\left\{\prod_{j=1}^{k_{i}^{\prime}} \delta\left(\left[v^{a_{\rho_{i}^{\prime}}-k_{i}^{\prime}+j} \rho_{i}^{\prime}, v^{c_{j}^{(i)}} \rho_{i}^{\prime}\right]\right) \mid i=1, \ldots, k\right\} .
$$

Proof. The arguments of the proof follow the same lines as those in the proof of Theorem 4.11. We, therefore, omit the proof here.

Theorem 4.14 and Theorem 4.15 imply that there exists an injective mapping from $D\left(\rho_{1}, \rho_{2}, \ldots, \rho_{k} ; \sigma_{\text {cusp }}, c\left(\sigma_{\text {cusp }}\right)\right)$ into the set of induced representations of the form (4-4). Finally, it remains to show that this mapping is surjective.

Theorem 4.16. The map described above gives a bijective correspondence between the set $D\left(\rho_{1}, \rho_{2}, \ldots, \rho_{k} ; \sigma_{\text {cusp }}, c\left(\sigma_{\text {cusp }}\right)\right)$ and the set of induced representations of the form (4-4). 
Proof. Let $\sigma$ be the unique irreducible subrepresentation of the form (4-4), i.e.,

$$
\left(\prod_{i=1}^{k} \prod_{j=1}^{k_{i}} \delta\left(\left[v^{a_{\rho_{i}}-k_{i}+j} \rho_{i}, v^{b_{j}^{(i)}} \rho_{i}\right]\right)\right) \rtimes c^{\prime}\left(\sigma_{\text {cusp }}\right) .
$$

Since $\rho_{p} ¥ \rho_{q}$ for $p \neq q$, we have for any $l=1, \ldots, k$ the embedding

$$
\begin{aligned}
\sigma \hookrightarrow\left(\prod_{i \neq l} \prod_{j=1}^{k_{i}} \delta\left(\left[v^{a_{\rho_{i}}-k_{i}+j} \rho_{i}, v^{b_{j}^{(i)}} \rho_{i}\right]\right)\right) \\
\quad \rtimes \delta\left(\left[v^{a_{\rho_{l}}-k_{l}+l} \rho_{l}, v^{b_{l}^{(l)}} \rho_{l}\right], \ldots,\left[v^{a_{\rho_{l}}} \rho_{l}, v^{b_{k_{l}}^{(l)}} \rho_{l}\right] ; c^{\prime}\left(\sigma_{\text {cusp }}\right)\right) .
\end{aligned}
$$

Theorem 4.12 implies that $\delta\left(\left[v^{a_{\rho_{l}}-k_{l}+l} \rho_{l}, v^{b_{l}^{(l)}} \rho_{l}\right], \ldots,\left[v^{a_{\rho_{l}}} \rho_{l}, v^{b^{(l)}} \rho_{l}\right] ; c^{\prime}\left(\sigma_{\text {cusp }}\right)\right)$ is strongly positive. Since $l$ can be arbitrary, $\sigma$ always has positive unitary exponents in the Jacquet module with respect to the Levi subgroup $\mathrm{GL}_{n_{\rho_{1}}} \times \mathrm{GL}_{n_{\rho_{1}}} \times \cdots \times$ $\mathrm{GL}_{n_{\rho_{k}}} \times G_{n^{\prime}}$. Therefore, $\sigma$ is strongly positive.

Since any strongly positive representation in $R$ can be considered an element of $D\left(\rho_{1}^{\prime}, \rho_{2}^{\prime}, \ldots, \rho_{k}^{\prime} ; \sigma_{\text {cusp }}^{\prime}, c^{\prime}\left(\sigma_{\text {cusp }}^{\prime}\right)\right)$ for some $\rho_{i}^{\prime}$ and $\sigma_{\text {cusp }}^{\prime}$, we can extend the bijective mapping constructed in Theorem 4.16 to any strongly positive representation in $R$. In sum, let SP and LJ be as defined in Section 1. Then we have a bijective correspondence between SP and LJ.

Remark 4.17. The ideas used in this section for the results of GSpin groups can be applied to even special orthogonal groups. Let us also remark that it is easier to work with even special orthogonal groups than even GSpin groups due to the results of Ban [1999a; 1999b] and Jantzen [2006]. For example, in the case of even special orthogonal groups, the Weyl group actions on the simple roots and induced representations are studied in [Ban 1999a; 1999b] and Tadić's structure formula is constructed in [Jantzen 2006, Theorem 3.4] (see also [Jantzen and Liu 2014, Theorem 3.1]). Let us remark that the classification of discrete series representations of $\mathrm{SO}_{2 n}$ is first proved by C. Jantzen [2011] using the results for $O_{2 n}$ in [Mœglin 2002; Mœglin and Tadić 2002]. Our approach is different from [Jantzen 2011] and we generalize Matić's idea to the case of even special orthogonal groups.

\section{Applications}

It is easy to see that the strongly positive representations are special kinds of discrete series due to Casselman's square integrability criterion in [Kim 2009]. Furthermore, the strongly positive representations can be considered basic building blocks for discrete series representations (Theorem 5.1). The proof of the following embedding theorem is exactly the same as the case of odd GSpin groups, since the main idea of the proof depends on a slight variation of Casselman's square integrability criterion 
for even GSpin groups (Proposition 3.2 in [Kim 2009]). Hence the proof is omitted. Let us remark that this idea originally comes from the results for metaplectic groups by Matić and we adapt some proofs from [Matić 2012, Chapter 3] to our situation.

Theorem 5.1. Let $\sigma$ denote a discrete series representation of $G_{n}$. Then there exists an embedding of the form

$$
\sigma \hookrightarrow \delta\left(\left[v^{a_{1}} \rho_{1}, v^{b_{1}} \rho_{1}\right]\right) \times \delta\left(\left[v^{a_{2}} \rho_{2}, v^{b_{2}} \rho_{2}\right]\right) \times \cdots \times \delta\left(\left[v^{a_{r}} \rho_{r}, v^{b_{r}} \rho_{r}\right]\right) \rtimes \sigma_{\mathrm{sp}}
$$

where $a_{i} \leq 0, a_{i}+b_{i}>0$ and $\rho_{i} \in R_{\mathrm{GL}}$ is an irreducible unitary supercuspidal representation for $i=1, \ldots, r$ (we allow $r=0$ ); and $\sigma_{\mathrm{sp}} \in R$ is a strongly positive representation.

Theorem 5.1, together with our main result Theorem 4.16 giving the classification of strongly positive representations, imply the embedding

$$
\begin{aligned}
\sigma \hookrightarrow \delta\left(\left[v^{a_{1}} \rho_{1}, v^{b_{1}} \rho_{1}\right]\right) \times \cdots \times & \delta\left(\left[v^{a_{r}} \rho_{r}, v^{b_{r}} \rho_{r}\right]\right) \\
& \times\left(\prod_{i=1}^{k} \prod_{j=1}^{k_{i}} \delta\left(\left[v^{a^{\rho_{i}^{\prime}}-k_{i}+j} \rho_{i}^{\prime}, v^{b_{j}^{(i)}} \rho_{i}^{\prime}\right]\right)\right) \rtimes \sigma_{\text {cusp }} .
\end{aligned}
$$

where $a_{i}, b_{i}$ and $\rho_{i}$ are as in Theorem 5.1; $a_{\rho_{i}^{\prime}}, b_{j}^{(i)}, k_{i}$ and $\rho_{i}^{\prime}$ are as in Theorem 4.14; and $\sigma_{\text {cusp }}$ is an irreducible supercuspidal representation of $G_{n}$.

This embedding has an interesting application in the proof of the equality of $L$ functions from the Langlands-Shahidi method and Artin $L$-functions through local Langlands correspondence (see [Shahidi 2010] for the Langlands-Shahidi method). More precisely, in [Kim 2015a] we used the following filtration of admissible representations to prove the equality of $L$-functions in the case of GSpin groups:

$$
\text { supercuspidal } \subseteq \text { discrete series } \subseteq \text { tempered } \subseteq \text { admissible. }
$$

We first showed the equality of $L$-functions in the supercuspidal case. Then, the above embedding (5-1) was used to generalize that result to the case of discrete series representations. Finally, that result was generalized via Langlands classification and properties of tempered representations to the cases of tempered representations and admissible representations. Furthermore, the equality of $L$-functions also has an interesting application in the proof of the generic Arthur packet conjecture in the case of GSpin groups [Shahidi 2011]. This conjecture can be considered a local version of the generalized Ramanujan conjecture.

\section{Acknowledgements}

This paper is based on part of my thesis. I would like to express my deepest gratitude to my advisor, Professor Shahidi for his constant encouragement and help. I would also like to thank Professor Tadić and Professor Matić for answering many 
questions and for their suggestions. This paper could not be done without their help. I also thank Professor Mœglin for answering many questions including the question about the half integer conjecture (Lemma 4.1). Thanks are also due to Professors Goldberg, Asgari and Jantzen for very helpful comments. I would also like to thank the referee, who read the paper very carefully, corrected a number of errors and gave very helpful comments.

\section{References}

[Asgari 2002] M. Asgari, "Local $L$-functions for split spinor groups", Canad. J. Math. 54:4 (2002), 673-693. MR 2003i:11062 Zbl 1011.11034

[Ban 1999a] D. Ban, "Parabolic induction and Jacquet modules of representations of O $(2 n, F)$ ", Glas. Mat. Ser. III 34(54):2 (1999), 147-185. MR 2001m:22033 Zbl 0954.22013

[Ban 1999b] D. Ban, "Self-duality in the case of SO(2n, F)", Glas. Mat. Ser. III 34(54):2 (1999), 187-196. MR 2002a:22022 Zbl 0954.22012

[Bernstein and Zelevinsky 1977] J. Bernstein and A. V. Zelevinsky, "Induced representations of reductive p-adic groups. I”, Ann. Sci. École Norm. Sup. (4) 10:4 (1977), 441-472. MR 58 \#28310 Zbl 0992.22015

[Bernstein et al. 1986] J. Bernstein, P. Deligne, and D. Kazhdan, "Trace Paley-Wiener theorem for reductive $p$-adic groups", J. Analyse Math. 47 (1986), 180-192. MR 88g:22016 Zbl 0634.22011

[Casselman 1995] W. Casselman, "Introduction to the theory of admissible representations of $p$-adic reductive groups", Lecture notes, 1995, Available at http://www.math.ubc.ca/ cass/research/pdf/ p-adic-book.pdf.

[Hanzer and Muić 2008] M. Hanzer and G. Muić, "On an algebraic approach to the Zelevinsky classification for classical p-adic groups”, J. Algebra 320:8 (2008), 3206-3231. MR 2009k:20107 Zbl 1166.22011

[Hundley and Sayag 2012] J. Hundley and E. Sayag, "Descent construction for GSpin groups", 2012. To appear in Mem. Amer. Math. Soc. arXiv 1110.6788

[Jantzen 2006] C. Jantzen, "Jacquet modules of induced representations for $p$-adic special orthogonal groups”, J. Algebra 305:2 (2006), 802-819. MR 2007m:22014 Zbl 1104.22019

[Jantzen 2011] C. Jantzen, "Discrete series for $p$-adic $\mathrm{SO}(2 n)$ and restrictions of representations of O(2n)”, Canad. J. Math. 63:2 (2011), 327-380. MR 2012f:22030 Zbl 1219.22016

[Jantzen and Liu 2014] C. Jantzen and B. Liu, "The generic dual of $p$-adic split $S O_{2 n}$ and local Langlands parameters”, Israel J. Math. 204:1 (2014), 199-260. MR 3273456 Zbl 1303.22008

[Kim 2009] W. Kim, "Square integrable representations and the standard module conjecture for general spin groups”, Canad. J. Math. 61:3 (2009), 617-640. MR 2010e:11048 Zbl 1258.11062

[Kim 2015a] Y. Kim, "Langlands-Shahidi $L$-functions for GSpin groups and the generic Arthur packet conjecture”, preprint, 2015. arXiv 1507.07156

[Kim 2015b] Y. Kim, "Strongly positive representations of GSpin $2 n+1$ and the Jacquet module method", Math. Z. 279:1-2 (2015), 271-296. MR 3299853 Zbl 06393879

[Matić 2011] I. Matić, "Strongly positive representations of metaplectic groups", J. Algebra 334 (2011), 255-274. MR 2012d:20011 Zbl 1254.22010

[Matić 2012] I. Matić, "Theta lifts of strongly positive discrete series: the case of $(\widetilde{\mathrm{Sp}}(n), O(V))$ ", Pacific J. Math. 259:2 (2012), 445-471. MR 2988500 Zbl 1277.22017 
[Mœglin 2002] C. Mœglin, "Sur la classification des séries discrètes des groupes classiques $p$-adiques: paramètres de Langlands et exhaustivité", J. Eur. Math. Soc. 4:2 (2002), 143-200. MR 2003g:22021 Zbl 1002.22009

[Mœglin 2014] C. Mœglin, "Paquets stables des séries discrètes accessibles par endoscopie tordue; leur paramètre de Langlands", pp. 295-336 in Automorphic forms and related geometry: assessing the legacy of I. I. Piatetski-Shapiro, edited by J. W. Cogdell et al., Contemp. Math. 614, American Mathematical Society, Providence, RI, 2014. MR 3220932 Zbl 1298.22019

[Mœglin and Tadić 2002] C. Mœglin and M. Tadić, "Construction of discrete series for classical p-adic groups", J. Amer. Math. Soc. 15:3 (2002), 715-786. MR 2003g:22020 Zbl 0992.22015

[Muić 2006] G. Muić, "On the non-unitary unramified dual for classical p-adic groups", Trans. Amer. Math. Soc. 358:10 (2006), 4653-4687. MR 2007j:22029 Zbl 1102.22014

[Shahidi 1990] F. Shahidi, "A proof of Langlands' conjecture on Plancherel measures; complementary series for p-adic groups", Ann. of Math. (2) 132:2 (1990), 273-330. MR 91m:11095 Zbl 0780.22005

[Shahidi 2010] F. Shahidi, Eisenstein series and automorphic L-functions, American Mathematical Society Colloquium Publications 58, American Mathematical Society, Providence, RI, 2010. MR 2012d:11119 Zbl 1215.11054

[Shahidi 2011] F. Shahidi, "Arthur packets and the Ramanujan conjecture", Kyoto J. Math. 51:1 (2011), 1-23. MR 2784745 Zbl 1238.11060

[Silberger 1980] A. J. Silberger, "Special representations of reductive $p$-adic groups are not integrable”, Ann. of Math. (2) 111:3 (1980), 571-587. MR 82k:22015 Zbl 0437.22015

[Tadić 1995] M. Tadić, "Structure arising from induction and Jacquet modules of representations of classical p-adic groups”, J. Algebra 177:1 (1995), 1-33. MR 97b:22023 Zbl 0874.22014

[Tadić 1998] M. Tadić, “On reducibility of parabolic induction”, Israel J. Math. 107 (1998), 29-91. MR 2001d:22012 Zbl 1166.22011

[Zelevinsky 1980] A. V. Zelevinsky, "Induced representations of reductive $p$-adic groups. II: On irreducible representations of GL(n)", Ann. Sci. École Norm. Sup. (4) 13:2 (1980), 165-210. MR 83g:22012 Zbl 0441.22014

Received March 10, 2015. Revised April 30, 2015.

YEANSU KIM

DEPARTMENT OF MATHEMATICS

UNIVERSITY OF IOWA

14 MACLEAN HALL

IOWA CITY, IA 52242

UNITED STATES

yeansu-kim@uiowa.edu 


\title{
PACIFIC JOURNAL OF MATHEMATICS
}

\author{
msp.org/pjm
}

Founded in 1951 by E. F. Beckenbach (1906-1982) and F. Wolf (1904-1989)

\section{EDITORS}

Don Blasius (Managing Editor)

Department of Mathematics

University of California

Los Angeles, CA 90095-1555

blasius@math.ucla.edu

\author{
Paul Balmer \\ Department of Mathematics \\ University of California \\ Los Angeles, CA 90095-1555 \\ balmer@math.ucla.edu \\ Robert Finn \\ Department of Mathematics \\ Stanford University \\ Stanford, CA 94305-2125 \\ finn@math.stanford.edu \\ Sorin Popa \\ Department of Mathematics \\ University of California \\ Los Angeles, CA 90095-1555 \\ popa@math.ucla.edu
}

\author{
Vyjayanthi Chari \\ Department of Mathematics \\ University of California \\ Riverside, CA 92521-0135 \\ chari@math.ucr.edu \\ Kefeng Liu \\ Department of Mathematics \\ University of California \\ Los Angeles, CA 90095-1555 \\ liu@math.ucla.edu \\ Jie Qing \\ Department of Mathematics \\ University of California \\ Santa Cruz, CA 95064 \\ qing@ cats.ucsc.edu
}

\section{PRODUCTION}

Silvio Levy, Scientific Editor, production@msp.org

\section{SUPPORTING INSTITUTIONS}

ACADEMIA SINICA, TAIPEI

CALIFORNIA INST. OF TECHNOLOGY

INST. DE MATEMÁTICA PURA E APLICADA

KEIO UNIVERSITY

MATH. SCIENCES RESEARCH INSTITUTE

NEW MEXICO STATE UNIV.

OREGON STATE UNIV.

\author{
STANFORD UNIVERSITY \\ UNIV. OF BRITISH COLUMBIA \\ UNIV. OF CALIFORNIA, BERKELEY \\ UNIV. OF CALIFORNIA, DAVIS \\ UNIV. OF CALIFORNIA, LOS ANGELES \\ UNIV. OF CALIFORNIA, RIVERSIDE \\ UNIV. OF CALIFORNIA, SAN DIEGO \\ UNIV. OF CALIF., SANTA BARBARA
}

\author{
Daryl Cooper \\ Department of Mathematics \\ University of California \\ Santa Barbara, CA 93106-3080 \\ cooper@math.ucsb.edu \\ Jiang-Hua Lu \\ Department of Mathematics \\ The University of Hong Kong \\ Pokfulam Rd., Hong Kong \\ jhlu@maths.hku.hk \\ Paul Yang \\ Department of Mathematics \\ Princeton University \\ Princeton NJ 08544-1000 \\ yang@math.princeton.edu
}

These supporting institutions contribute to the cost of publication of this Journal, but they are not owners or publishers and have no responsibility for its contents or policies.

See inside back cover or msp.org/pjm for submission instructions.

The subscription price for 2016 is US $\$ 440 /$ year for the electronic version, and $\$ 600 /$ year for print and electronic.

Subscriptions, requests for back issues and changes of subscribers address should be sent to Pacific Journal of Mathematics, P.O. Box 4163, Berkeley, CA 94704-0163, U.S.A. The Pacific Journal of Mathematics is indexed by Mathematical Reviews, Zentralblatt MATH, PASCAL CNRS Index, Referativnyi Zhurnal, Current Mathematical Publications and Web of Knowledge (Science Citation Index).

The Pacific Journal of Mathematics (ISSN 0030-8730) at the University of California, c/o Department of Mathematics, 798 Evans Hall \#3840, Berkeley, CA 94720-3840, is published twelve times a year. Periodical rate postage paid at Berkeley, CA 94704, and additional mailing offices. POSTMASTER: send address changes to Pacific Journal of Mathematics, P.O. Box 4163, Berkeley, CA 94704-0163.

PJM peer review and production are managed by EditFLOW ${ }^{\circledR}$ from Mathematical Sciences Publishers.

\section{PUBLISHED BY}

\section{mathematical sciences publishers \\ nonprofit scientific publishing}

http://msp.org/

(C) 2016 Mathematical Sciences Publishers 


\section{PACIFIC JOURNAL OF MATHEMATICS}

Volume $280 \quad$ No. $1 \quad$ January 2016

Stable capillary hypersurfaces in a wedge

JAIGYOUNG CHOE and MIYUKI KoISO

The Chern-Simons invariants for the double of a compression body

DAVID L. DUNCAN

Compactness and the Palais-Smale property for critical Kirchhoff equations in

closed manifolds

EMMANUEL HeBEy

On the equivalence of the definitions of volume of representations

SUNGWOON KIM

Strongly positive representations of even GSpin groups

YEANSU KIM

An Orlik-Raymond type classification of simply connected 6-dimensional torus manifolds with vanishing odd-degree cohomology

\section{SHINTARÔ KUROKI}

Solutions with large number of peaks for the supercritical Hénon equation

Zhongyuan LiU and ShuangJie Peng

Effective divisors on the projective line having small diagonals and small heights and 141 their application to adelic dynamics

YÛSUKE OKUYAMA

Computing higher Frobenius-Schur indicators in fusion categories constructed from inclusions of finite groups

\section{Peter Schauenburg}

Chordal generators and the hydrodynamic normalization for the unit ball

SEBASTIAN SCHLEISSINGER

On a question of A. Balog

ILYA D. SHKREDOV

Uniqueness result on nonnegative solutions of a large class of differential inequalities 241 on Riemannian manifolds

YUHUA SUN

Correction to "Closed orbits of a charge in a weakly exact magnetic field"

WILL J. MERRY 